\section{Made to move in half the time}

Advances in the Invisalign system now enable dental practitioners to offer their patients one-week aligner wear instead of previously recommended two-week changes, allowing shorter treatment time. This means that straighter teeth can now be achieved in as little as three months.

The Invisalign system is designed for straightening teeth with a series of virtually invisible, clear plastic aligners which are custom-made for each patient. The discretion and effectiveness of the system has made it highly desirable, appealing to millions of patients who have been successfully treated with Invisalign treatment.

Until now, the custom-made aligners were always recommended to be changed every two weeks to incrementally move patients' teeth towards their new position. However, recent technological advancement now allows Align

Technology to recommend one-week aligner wear, provided their case is deemed suitable by their provider. This switch from two to one-week wear is the result of 15 years of perpetual product innovation, which has culminated in the combined use of new materials, features and technology.

Because of this, weekly aligner changes may reduce treatment time up to $50 \%$ compared with two-week changes, which means patients who are deemed suitable for one week aligners, depending on their treatment option - Invisalign Lite and/or Invisalign Go - could see their treatment through to completion in as little as three months.

This means that for people looking to make a change in the appearance of their teeth before a wedding or another milestone event, it may be possible to see straighter teeth and make it in time for the big day.

First steps would be to consult their Invisalign provider who would prescribe the most suitable treatment option, as well as advise on the time required for their treatment.

For more information visit www. invisalign.co.uk.

\section{High quality, top results}

For successful endodontic treatments, it's essential that you use high quality products. ROEKO Guttapercha Points from Coltene are a perfect example.

Used in combination with sealers, Guttapercha Points are used to effectively obturate the root canal after endodontic treatments. Available in different ISO sizes, in different tapers and with a top colour coding system, they are the ideal option for top endodontic results.

What's more, Guttapercha Points are easy to introduce to the canal and are stiff yet flexible enough to follow the curves of the canal without breakage. They also exhibit excellent tissue compatibility and are absolutely hygienic for added peace of mind.

Coltene's fully automated production system also ensures that each and every item is finished to exacting standards, to ensure you are never let down.

In endodontics, if you want top results you must use high quality materials. To find out more about ROEKO Guttapercha Points, contact the Coltene team today.

To find out more about Coltene products, visit www.coltene.com.

\section{Don't get derailed by the details}

In dentistry, making sure you've got the details just right can be the difference between a good restoration and a great one.

That's why practitioners who have a real eye for the details use Imprint 4 impression material from 3M Oral Care. Let's take a look:

- Advanced hydrophilicity and flow properties for ease of use

- Ability to capture fine details

- Unique self-warming technology, resulting in a low snap set time and reduced drag

All of this allows you to create accurately fitting restorations with less effort and risk of retakes.

Don't get derailed by the details. Find out more by contacting the $3 \mathrm{M}$ Oral Care team today. Call 08456025094 or visit www.3Mespe.co.uk.

$3 \mathrm{M}$ and Imprint are trademarks of the $3 \mathrm{M}$ Company

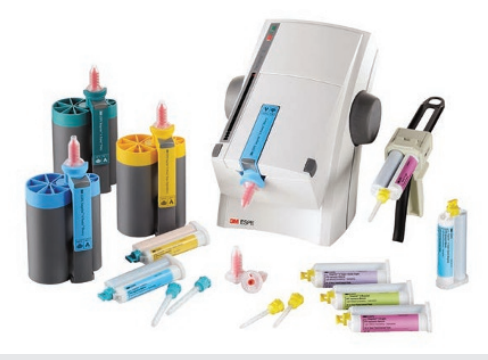

\title{
The perfect adjunctive solution
}

The Waterpik Water Flosser provides the perfect adjunctive product for all your patients to use, regardless of whether they have crowns, bridges, implants or orthodontic appliances.

The innovative solution has been shown to be up to $50 \%$ more effective at reducing gingivitis and up to $93 \%$ more effective for reducing bleeding compared to string floss, as well as more effective than interdental brushes.

The latest product - the Waterpik Cordless Advanced - is a rechargeable model and offers further advantages as its compact design, waterproof casing and travel plug make it ideal for use in small bathrooms or for patients on-the-go.

Find out more about the innovative Waterpik Water Flosser by calling them on 03331235677.

For more information visit www.waterpik. co.uk. Waterpik products are available from Amazon, Costco UK and Superdrug stores across the UK and Ireland. 